\title{
O Código Florestal tem Base Científica?
}

\author{
Jean Paul Metzger*
}

Departamento de Ecologia, Universidade de São Paulo - USP, São Paulo, SP, Brasil

\section{Introdução}

Existem muitas dúvidas sobre qual foi o embasamento científico que permitiu definir os parâmetros e os critérios da lei 4.771/65 de 15 de Setembro de 1965, mais conhecida como Código Florestal. Dentre estas dúvidas, podemos incluir as bases teóricas que permitiram definir:

i) as larguras das Áreas de Preservação Permanente (APP);

ii) a extensão das Reservas Legais (RL) nos diferentes biomas brasileiros;

iii) a necessidade de se separar RL da APP, e de se manter RL com espécies nativas; e

iv) a possibilidade de se agrupar as RL de diferentes proprietários em fragmentos maiores.

Neste artigo, eu procuro analisar estas questões, tentando entender se os avanços da ciência nos últimos 45 anos permitem, ou não, sustentar o Código Florestal de 1965 e suas modificações ocorridas posteriormente. Esse trabalho não tem por objetivo fazer uma compilação completa de trabalhos científicos relacionados ao Código Florestal, objetivo esse que demandaria um tempo e esforço muito mais amplo. Dada a minha especialidade, eu vou me limitar à discussão dos quatro pontos acima, para os quais a ecologia tem importantes contribuições. Ademais, eu me ative a trabalhos feitos em ecossistemas brasileiros, para considerar a complexidade e as particularidades destes sistemas. Limitei também a busca a trabalhos com amplo respaldo internacional, dando assim preferência a artigos publicados em revistas científicas internacionais e/ou compilados pelos sistemas Scopus (http://www.scopus.com/) ou ISI Web of Knowledge (http://apps.isiknowledge.com/).

\footnotetext{
*Send correspondence to: Jean Paul Metzger

Departamento de Ecologia, Universidade de São Paulo - USP,

São Paulo, SP, Brasil

E-mail: jpm@ib.usp.br
}

\section{Qual a Extensão Mínima das Áreas de Preservação Permanente?}

O Código Florestal estipula uma série de larguras mínimas de áreas de proteção ao longo de cursos d'água, reservatórios e nascentes. Qual foi a base científica usada para definir que corredores ripários deveriam ter no mínimo $30 \mathrm{~m}$ de proteção ao longo de cada margem do rio (além do limite das cheias anuais)? Será que essa largura não deveria variar com a topografia da margem, com o tipo de solo, com o tipo de vegetação, ou com o clima, em particular com a pluviosidade local?

A efetividade destas faixas de vegetação remanescente certamente depende de uma série de fatores, dentre eles o tipo de serviço ecossistêmico considerado e a largura de vegetação preservada. Por exemplo, há dados que indicam que larguras de $30 \mathrm{~m}$ seriam suficientes para as matas ripárias retirarem da água do lençol freático boa parte dos nitratos vindos dos campos agrícolas (Pinay \& Décamps 1988). No entanto, dada suas múltiplas funções, incluindo a fixação de solo, proteção de recursos hídricos e conservação de fauna e flora, deve-se pensar na largura mínima suficiente para que esta faixa desempenhe de forma satisfatória todas as suas funções. Por consequência, a definição desta largura no âmbito do Código Florestal deveria respeitar a função mais exigente. Eu não pretendo aqui fazer uma ampla revisão sobre a influência da largura das APP, mas penso que a conservação da biodiversidade possa ser um dos fatores mais limitantes para a definição de larguras mínimas, e por isso foquei minha revisão neste aspecto, dando ênfase ao caso das matas ripárias.

Em termos biológicos, os corredores são reconhecidos como elementos que facilitam o fluxo de indivíduos ao longo da paisagem. Em paisagens fragmentadas, quando o habitat original encontra-se disperso em inúmeros fragmentos, isolando e reduzindo o tamanho das populações nativas, a sobrevivência das espécies depende de suas habilidades de se deslocarem pela paisagem. Nestas condições, os corredores podem ter papel capital, pois muitas espécies não conseguem usar ou cruzar áreas abertas criadas pelo homem, nem quando se trata de áreas muito estreitas como estradas (Develey \& Stouffer 2001), e a existência 
de uma continuidade na cobertura vegetacional original é assim essencial. Dentre os benefícios dos corredores, já comprovados por pesquisa no Brasil, estão o aumento da diversidade genética (Almeida Viera \& De Carvalho 2008), o aumento da conectividade da paisagem, possibilitando o uso de vários pequenos fragmentos remanescentes de habitat, que isoladamente não sustentariam as populações (Awade e Metzger 2008; Boscolo et al. 2008; Martensen et al. 2008), a amenização dos efeitos da fragmentação (Pardini et al. 2005), e o potencial de amenizar os impactos de mudanças climáticas, numa escala temporal mais ampla (Marini et al. 2009).

A importância de florestas ripárias foi evidenciada em diferentes biomas brasileiros, e para diferentes grupos taxonômicos. A maior parte dos estudos foi feita na Floresta Atlântica (Metzger et al. 1997; Uezu et al. 2005; Marinho-Filho \& Verissimo 2007; Keuroghlian \& Eaton 2008; Maltchik et al. 2008; Martensen et al. 2008), mas existem dados também para Floresta Amazônica (Lima \& Gascon 1999; Michalski et al. 2006; Lees \& Peres 2008), Caatinga (Moura \& Schlindwein 2009), Pantanal (Quigley \& Crawshaw 1992) e Cerrado (Tubelis et al. 2004). Em relação aos grupos taxonômicos, há dados para árvores (Metzger et al. 1997), anfíbios (Lima e Gascon 1999; Maltchik et al. 2008), aves (Tubelis et al. 2004; Uezu et al. 2005; Martensen et al. 2008), grandes mamíferos (Quigley \& Crawshaw 1992; Marinho-Filho \& Verissimo 2007; Keuroghlian \& Eaton 2008; Lees \& Peres 2008), pequenos mamíferos (Lima \& Gascon 1999) e abelhas (Moura e Schlindwein 2009). Não há dúvidas que independentemente do bioma ou do grupo taxonômico considerado, toda paisagem deveria manter corredores ripários, dado os seus benefícios para a conservação das espécies.

Os benefícios dos corredores podem estar relacionados à largura, extensão, continuidade e qualidade dos corredores (Laurance e Laurance 1999), à topografia e largura das áreas de influência ripária (Metzger et al. 1997), entre outros fatores, mas sem dúvida o fator mais importante é a largura. Esta largura afeta a qualidade do habitat, regulando a área impactada pelos efeitos de borda, i.e. pelas as modificações micro-climáticas e pelo aumento das perturbações que ocorrem nas bordas destes habitats. Em ambiente florestal, há aumento da luminosidade e do ressecamento do ar e do solo, além de um aumento na entrada de espécies invasoras e generalistas (vindas de áreas antrópicas), e de perturbações ocasionais (rajadas de vento, queimadas) que excluem algumas espécies nativas, mais especializadas em sombra, e levam a uma maior mortalidade. Esses efeitos de borda podem variar em extensão em função das espécies e dos processos considerados, e também de acordo com as características físicas do local, em particular com a orientação solar, a latitude e o tipo de matriz de ocupação adjacente, que influenciam na quantidade de radiação solar incidente. De uma forma geral, os efeitos mais intensos ocorrem nos 100 primeiros metros (Laurance et al. 2002), o que implica que corredores com menos de 200 m são formados essencialmente por ambientes de borda, altamente perturbados. Assim, alguns autores sugerem que corredores estreitos perderiam parte de sua utilidade, por favorecerem unicamente espécies generalistas, que suportam os efeitos de borda (Santos et al. 2008; Lopes et al. 2009). Espécies mais estritamente florestais necessitariam de corredores de pelo menos $200 \mathrm{~m}$ de largura (Laurance \& Laurance 1999; Lees e Peres 2008).

Trabalhos que consideraram a funcionalidade biológica dos corredores em função da largura indicam valores mínimos superiores a $100 \mathrm{~m}$. Na Amazônia, larguras de 140 a 190 m são necessárias para haver certa similaridade entre as comunidades de pequenos mamíferos e de anfíbios de serapilheira entre elementos florestais lineares e uma área controle de floresta contínua (Lima e Gascon 1999). Ainda na Amazônia, Lee \& Peres (2008) recensearam aves e mamíferos em 32 corredores, e observaram que a acumulação de espécies ocorreu até $400 \mathrm{~m}$ de largura para os dois grupos. A partir desse conjunto de dados, que devem representar situações encontradas em outras regiões da Amazônia, os autores sugerem que as APP ao longo de rios deveriam manter pelo menos $200 \mathrm{~m}$ de área florestada de cada lado do rio para que haja uma plena conservação da biodiversidade. A manutenção de corredores de $60 \mathrm{~m}$ (30 m de cada lado do rio), conforme a legislação atual, resultaria na conservação de apenas $60 \%$ das espécies locais. No Cerrado, Tubelis et al. (2004) sugerem que as matas de galeria tenham pelos menos $120 \mathrm{~m}$ de largura para a devida proteção das aves. Na Mata Atlântica, Metzger et al. (1997, 1998) trabalharam com 15 corredores de mata ripária ao longo do rio Jacaré-Pepira, no interior do estado de São Paulo. Nestes corredores, que variaram de 30 a $650 \mathrm{~m}$ de largura, os autores levantaram a diversidade de árvores e arbustos, e puderam observar que apenas 55\% delas estava presente em corredores de menos de $50 \mathrm{~m}$, enquanto $80 \%$ estava presente em corredores com mais $100 \mathrm{~m}$. Esses dados confirmam que corredores de apenas $30 \mathrm{~m}$ têm capacidade muito limitada de manutenção da biodiversidade.

Desta forma, o conhecimento científico obtido nestes últimos anos permite não apenas sustentar os valores indicados no Código Florestal de 1965 em relação à extensão das Áreas de Preservação Permanente, mas na realidade indicam a necessidade de expansão destes valores para limiares mínimos de pelos menos $100 \mathrm{~m}$ (50 m de cada lado do rio), independentemente do bioma, do grupo taxonômico, do solo ou do tipo de topografia.

\section{Qual a Quantidade Mínima de RL em Termos de Conservação de Biodiversidade?}

A extensão das Reservas Legais varia entre biomas, sendo mais ampla na Amazônia, e mais restrita em outras regiões do Brasil. Há dados científicos que permitam sustentar os valores de 20,35 e $80 \%$ de RL? 
$\mathrm{O}$ adequado debate dessas questões necessita considerar, antes de mais nada, a função das RL. Apesar de inicialmente essas reservas terem sido planejadas como reservas de “exploração florestal", elas são hoje em dia consideradas, segundo o Código Florestal, como áreas voltadas ao:

[...] uso sustentável dos recursos naturais, à conservação e reabilitação dos processos ecológicos, à conservação da biodiversidade e ao abrigo e proteção de fauna e flora nativas (Código Florestal).

Trata-se, basicamente, de elementos da paisagem que deveriam promover ou auxiliar a conservação da biodiversidade.

Neste âmbito, a definição da extensão das RL poderia ser pautada, teoricamente, em questões relacionadas com Populações Mínimas Viáveis, ou com áreas mínimas para se manter populações viáveis de grande predadores. Infelizmente, as evidências empíricas descartam a existência de um valor único, válido para todas as populações e comunidades, e apontam para áreas muito extensas para se conservar a integridade de um sistema ecológico (Soulé \& Simberloff 1986). Esta literatura é certamente útil para definição das áreas das Unidades de Conservação, mas é de pouco valor no caso das RL. Por outro lado, há um conjunto de dados e teorias, mais recentes, que são de grande valia nesta questão: os limiares de percolação e de fragmentação.

O limiar de percolação é a quantidade mínima de habitat necessária numa determinada paisagem para que uma espécie, que não tem capacidade de sair do seu habitat, possa cruzar a paisagem de uma ponta a outra. A teoria da percolação foi desenvolvida inicialmente na física, para solucionar questões sobre a quantidade mínima de material condutor necessário para prover condutividade elétrica, e agora é amplamente utilizada em ecologia para questões de conectividade biológica. Em simulações feitas em computador, foi possível definir o limiar de percolação como sendo de 59,28\% em paisagens aleatórias, homogêneas (Stauffer 1985). Acima deste valor, o habitat encontra-se ainda mais agrupado, em grandes fragmentos, favorecendo os fluxos biológicos pela paisagem, inclusive de espécies que não se deslocam fora do seu habitat. No limiar, há uma mudança brusca na estrutura da paisagem, com redução no tamanho dos fragmentos, aumento no número e no isolamento dos fragmentos, e logo perda repentina da conectividade da paisagem. Isso resulta em paisagens fragmentadas, com baixa capacidade de manter diversidade biológica (Metzger \& Décamps 1997). Apesar deste valor ter sido definido para paisagens aleatórias, estudos considerando três padrões distintos de fragmentação na Amazônia sustentam a ocorrência de mudanças bruscas em valores próximos a 60\% (Oliveira-Filho \& Metzger 2006). Na realidade, ocorrem mudanças estruturais bruscas em diferentes momentos. Em particular, há uma perda brusca no tamanho médio dos fragmentos por volta de 70 a $80 \%$ de habitat remanescente, além do esperado aumento do isolamento, da fragmentação e redução da conectividade para valores intermediários (30 a 60\%). Todas essas modificações levam a uma redução na capacidade da paisagem de sustentar diversidade biológica. Esse conjunto de dados indica a necessidade de se manter 60 a $70 \%$ do habitat original para que a paisagem tenha uma estrutura adequada para fins de conservação. Valores mais baixos de cobertura nativa ainda poderiam resultar em estruturas favoráveis para conservação, mas isso unicamente no caso de haver forte agregação deste habitat (Metzger 2001). Porém, como o controle sobre a agregação das RL não é uma tarefa fácil em termos operacionais, esta opção não deveria ser considerada. Na Amazônia, onde temos um vasto patrimônio biológico e genético ainda pouco conhecido, e relativamente conservado, dever-se-ia manter paisagens com pelo menos $60 \%$ de cobertura (Metzger 2002), ou de preferência com mais de $70 \%$, para se evitar os efeitos iniciais da redução brusca do tamanho dos fragmentos. Essas paisagens poderiam permear as Unidades de Conservação e as Terras Indígenas, facilitando desta forma o fluxo de boa parte das espécies entre estas unidades, contribuindo para a conservação da biodiversidade numa escala regional Se a extensão das APP estiver entre 10 a $20 \%$, como apontam dados preliminares de Miranda et al. (2008), as RL deveriam ser de pelo menos $50 \%$, e preferencialmente mais de $60 \%$. Os valores estipulados atualmente pelo Código Florestal para a Amazônia são um pouco mais altos (80\%, incluindo as APP), e podem ser justificados pelo princípio de precaução, dada à imensa riqueza biológica encontrada nestes sistemas, pelo conhecimento ainda restrito sobre os efeitos em longo prazo do desmatamento na Amazônia, e pelas amplas possibilidades de exploração sustentável de produtos florestais.

Em outras regiões mais intensamente ocupadas, onde a taxa de conversão de habitat nativo para uso humano foi mais intenso (e.g. na Mata Atlântica, no Cerrado, na Caatinga), esse limiar não poderia ser aplicado, a não ser que se pense em amplas ações de restauração. Porém, nesses casos de maior perda da cobertura nativa, há um outro conjunto de dados, que surgiu nos últimos vinte anos, que permite avaliar a extensão da RL: trata-se do limiar de fragmentação (Andrén 1994; Fahrig 2003). Segundo revisões feitas por esses autores, baseadas essencialmente em espécies de áreas temperadas, existiria um limiar de cobertura de habitat abaixo do qual os efeitos da fragmentação (i.e., da sub-divisão do habitat) se somariam aos efeitos da perda do habitat. Assim, acima deste limiar, que em geral é indicado por volta de $30 \%$ de habitat remanescente, os efeitos sobre a redução populacional ou a perda de diversidade biológica seriam principalmente devido à perda do habitat, enquanto que abaixo deste limiar haveria também um efeito forte da distribuição espacial do habitat, em particular de sua sub-divisão. Esse limiar não é unânime e nem sempre há suporte empírico para ele, como têm demonstrados alguns resultados obtidos em zona tropical que relatam efeitos de fragmentação ao longo de todo o processo de perda de habitat (Develey \& Metzger 2006), ou então que 
indicam que esse limiar pode variar em função do grupo de organismos considerados, em particular em função da sensibilidade deles à perda de habitat (Lindenmayer \& Luck 2005). Porém, há claras evidências, inclusive obtidas recentemente no Brasil, que paisagens com menos de $30 \%$ de habitat tendem a ter apenas fragmentos pequenos e muito isolados, e suportam por conseqüência comunidades muito empobrecidas, e isso para diferentes grupos taxonômicos (Martensen et al. 2008; Metzger et al. 2009). O limiar de 30\% poderia ser considerado, assim, como um limite mínimo de cobertura nativa que uma paisagem intensamente utilizada pelo homem deveria ter, permitindo conciliar uso econômico e conservação biológica. Dado que as estimativas de porcentagem de APP variam para a grande maioria dos estados brasileiros de 10 a 20\% do território (Miranda et al. 2008), já excluindo as Unidades de Conservação (inclusive as de Uso Sustentável) e Terras Indígenas, o valor de 20\% para RL permitiria manter, na maioria dos casos, uma cobertura acima deste limiar.

Desta forma, a literatura sobre limiares em ecologia sustenta a definição de limites mínimos de RL de 50\% ou preferencialmente $60 \%$ na Amazônia, e de pelo menos $20 \%$ em regiões mais intensamente ocupadas, isso sem incluir as APP nestes percentuais.

\section{Reserva Legal: Sua Função Pode Ser Mantida com a Incorporação das APP ou com o Uso de Espécies Exóticas?}

Há fortes pressões para se flexibilizar o Código Florestal, no intuito principal de facilitar a expansão econômica e a regularização de atividades agrícolas, e isso poderia ser obtido por duas formas:

i) a inclusão das APP no cômputo das RL; e

ii) o uso de espécies de interesse econômico, em geral exóticas, numa parte destas reservas.

Mais uma vez, a questão levantada aqui é de saber quais são as bases científicas para essas mudanças.

A inclusão das Áreas de Preservação Permanente no cômputo da Reserva Legal já é prevista no Código Florestal, podendo ocorrer para todas as propriedades em áreas florestadas da Amazônia Legal, ou então quando APP e RL somam $50 \%$ ou mais da propriedade nas demais regiões do Brasil (ou seja, quando as APP cobrem mais de $30 \%$ da propriedade), ou $25 \%$ no caso das propriedades pequenas, que são aquela com 30 ou 50 ha, em função da localização no país. A questão é de saber se a inclusão da APP no cômputo da RL pode ser generalizada, ao invés de ocorrer apenas nas três situações mencionadas acima. Esta ampla inclusão é defendida por aqueles que consideram insuficientes as áreas disponíveis atualmente para expansão agrícola, urbana ou industrial (Miranda et al. 2008). Por outro lado, essa inclusão é rebatida de diversas formas, sendo o argumento mais comum o fato de ca. 3 milhões de $\mathrm{km}^{2}$ serem área mais do que suficiente para a expansão das atividades econômicas, além da existência de amplas áreas já utilizadas, mas que se encontram degradadas, e que deveriam ser alvo de projetos de recuperação para futura exploração. Esses argumentos são sem dúvida pertinentes, porém eu gostaria de acrescentar ao debate uma outra linha de raciocínio, apresentada a seguir.

Como dito anteriormente, as RL visam essencialmente à conservação da biodiversidade e ao uso sustentável de recursos naturais, enquanto as APP têm como:

[...] função ambiental de preservar os recursos hídricos, a paisagem, a estabilidade geológica, a biodiversidade, $o$ fluxo gênico de fauna e flora, proteger o solo e assegurar o bem-estar das populações humanas" (artigo primeiro do Código Florestal).

As APP basicamente evitam a erosão de terrenos declivosos e a colmatagem dos rios, asseguram os recursos hídricos, propiciam fluxo gênico, e prestam assim serviços ambientais capitais. Certamente essas áreas também contribuem para a conservação da biodiversidade, porém considerá-las equivalentes às RL seria um grande erro. Por se situarem justo adjacentes às áreas ripárias, em terrenos declivosos, ou ainda em restingas, tabuleiros, chapadas, e em áreas elevadas (acima de $1.800 \mathrm{~m}$ de altitude), as APP apresentam embasamento geológico e pedológico, clima e dinâmica hidro-geomorfológica distintas daquelas situadas distantes dos rios, em terrenos planos, mais longe das influências marinhas, ou em altitudes mais baixas. Em conseqüência disso, a composição de espécies da flora e da fauna nativa varia enormemente quando se comparam áreas situadas dentro e fora das APP. As evidências mais claras destas variações foram obtidas ao longo dos rios, mostrando, em particular, que a composição arbórea muda em função da distância ao leito do rio, sendo que as diferenças mais bruscas são obtidas nos primeiros 10-20 m (Oliveira-Filho 1994a,b; Metzger et al. 1997; Rodrigues \& Leitão-Filho 2004). Ou seja, as APP não protegem as mesmas espécies presentes nas RL, e vice-versa. Em termos de conservação biológica, essas áreas se complementam, pois são biologicamente distintas, e seria um grande erro ecológico considerá-las como equivalentes. Todo planejamento territorial deveria considerar a heterogeneidade biológica, e um dos primeiros passos neste sentido é distinguir RL e APP, mantendo estratégias distintas para a conservação nestas duas situações.

O segundo mecanismo de flexibilização das RL também já está parcialmente contemplado no Código Florestal, uma vez que em:

[...] pequenas propriedades ou de posse rural familiar, podem ser computados os plantios de árvores frutíferas ornamentais ou industriais, compostos por espécies exóticas, cultivadas em sistema intercalar ou em consórcio com espécies nativas (terceiro parágrafo do artigo 16). 
O que se discute, mais recentemente, é a ampliação desta flexibilização, permitindo que até $50 \%$ da RL possa ser composta por espécies exóticas, como o dendê ou o Eucalipto. Qual seria a efetividade da RL em termos de conservação biológica neste caso? Creio que já temos dados concretos para responder essa pergunta, em particular vindos de estudos de sistemas consorciados na Bahia, e de plantações de Eucalipto na Amazônia e na Mata Atlântica. Na região de Ilhéus, um grupo de pesquisadores das Universidades Estaduais de Campinas, São Paulo e Santa Cruz estudou o valor, em termos de conservação, de um sistema denominado "cabruca", que são plantações de cacau sombreadas por um dossel de mata (Faria et al. 2006, 2007; Pardini et al. 2009). A principal conclusão que esses pesquisadores chegaram é que o valor da cabruca depende do contexto no qual ela se encontra. Em paisagens predominantemente florestais, com amplas extensões de florestas maduras (ca. 50\%), e também com presença de manchas de florestas secundárias (16\%) e áreas produtivas florestadas (no caso, cabrucas, que cobrem $6 \%$ da paisagem, e seringais), as cabrucas conseguem manter uma parcela considerável das comunidades estudadas (samambaia, sapos, lagartos, morcegos e aves). No entanto, em outra paisagem vizinha, na qual as cabrucas dominam a paisagem (ca. 82\%), e os remanescentes florestais são reduzidos (ca. 5\%) e fragmentados, estes sistemas são extremamente emprobrecidos, e mantêm uma parcela pequena da biodiversidade regional (Faria et al. 2006, 2007). Ou seja, a ocorrência ou manutenção da fauna e flora nativa em cabrucas depende da existência de uma fonte de espécie próxima relativamente extensa. Isso significa que em paisagens predominantemente florestais, tais quais as que se quer conservar na Amazônia, sistemas similares ao das cabrucas poderiam ser considerados como boas alternativas de uso sustentável de recursos naturais em parte da RL (sendo que a extensão destas áreas deve ser estudada com cuidado). No entanto, em outras regiões do Brasil, onde a vegetação nativa já está consideravelmente reduzida e fragmentada, RL formadas por sistemas que intercalam espécies plantadas de interesse econômico com espécies nativas teriam reduzido valor conservacionista, $\mathrm{e}$ esta opção deveria ser evitada.

No caso das plantações de espécies de uso comercial, em geral exóticas, como o Eucalipto, a situação é distinta. Estudos promovidos no Rio Grande do Sul mostram que estas monoculturas arbóreas podem conter parte da biota nativa, porém isso depende fortemente do tipo de manejo da plantação, e em particular da manutenção da regeneração de espécies nativas no sub-bosque, e da ligação das áreas plantadas com fontes de espécies nativas próximas (Fonseca et al. 2009). Infelizmente, a grande maioria dos reflorestamentos comerciais não segue essas regras. Num dos mais completos estudos sobre esses reflorestamentos feitos no país, no projeto Jarí (Amazônia), Barlow et al. (2007a, b) mostraram, para diferentes grupos taxonômicos, haver baixa similaridade de espécies entre florestas nativas maduras e áreas de reflorestamento, deixando claro o limitado valor destas plantações em conservar espécies nativas.
Logo, independentemente da cobertura florestal remanescente na paisagem, não é aconselhável a substituição de RL de espécies nativas por plantações homogêneas de espécies exóticas. Por outro lado, sistemas consorciados de espécies nativas e de interesse econômico podem ser opções interessantes para parte das RL da Amazônia, num contexto de ampla cobertura florestal nativa. Ademais, a fusão de APP e RL seria temerária em termos biológicos simplesmente porque estas têm funções e composições de espécies distintas, e desempenham assim papeis complementares em termos de conservação da biodiversidade.

\section{Devemos Manter Pequenos Fragmentos de Vegetação Nativa Sob Forma de RL?}

O valor de pequenos fragmentos de RL para a conservação da biodiversidade vem sendo questionado, levando a propostas de não mais contabilizar essas reservas por propriedade, mas sim por bacia hidrográfica ou mesmo por bioma, de forma a agrupar essas áreas em fragmentos maiores, e assim aumentar seu valor biológico. Esse mecanismo é conhecido como "regime de condomínio", e já foi inserido no Código Florestal. Essa opção de agregação das RL tem respaldo em ampla discussão ocorrida nas décadas de 1970 e 1980, que considerava duas opções principais de conservação: um único fragmento grande, ou vários pequenos fragmentos de área equivalente ao fragmento grande (em Inglês, "Single Large or Several Small", comumente denominada de SLOSS; (Simberloff \& Abele 1976, 1982; Diamond 1975, 1976)). Apesar desta questão não considerar fatores essenciais para uma devida comparação, em particular o tamanho dos fragmentos pequenos e o grau de isolamento entre eles, a discussão evidenciou que muitos fragmentos pequenos podem abrigar mais espécies do que um fragmento grande, por representarem áreas com características distintas, e logo com composições menos similares. Por outro lado, um fragmento grande é a melhor opção em termos de manutenção das espécies por longo prazo, pois fragmentos grandes contêm em geral populações maiores, que são assim mais resistentes a flutuações ambientais, demográficas ou genéticas (Shaffer 1987), além de serem menos impactados pelos efeitos de borda. Enfim, estratégias de conservação que permitam manter as espécies em longo prazo devem dar prioridade a grandes fragmentos, o que sustentaria a proposta de agregação de RL de diferentes propriedades numa única área.

Os benefícios desta estratégia dependem também da representatividade biológica da rede de RL, e da manutenção de paisagens permeáveis entre os grandes núcleos de conservação da biodiversidade, que são as Unidades de Conservação de proteção integral. Ou seja, a efetividade do agrupamento de RL em fragmentos grandes depende destes fragmentos representarem comunidades biológicas similares àquelas que estariam presentes nos pequenos fragmentos de RL. Caso isso não ocorra, há grandes riscos de extinção de espécies características das áreas mais propícias para uso econômico, mantendo apenas a biota de áreas menos propícias ao uso (e.g., solos pobres ou pedregosos, ou terrenos em áreas íngremes). Em casos extremos, se a 
compensação puder ser feita em qualquer região de um mesmo bioma, pode haver extinção das espécies presentes na Mata Atlântica da Bahia, e manutenção apenas daquelas presentes na Serra do Mar. Esta situação certamente não seria desejável, uma vez que estas áreas não são equivalentes, pois possuem condições ambientais e histórias evolutivas distintas, e logo têm composições de espécies distintas, sendo ambas relevantes em termos de conservação.

Ademais, a concentração excessiva de RL numa única região, mesmo que situada em áreas biologicamente equivalentes, poderia levar a existência de desertos biológicos, formados por amplas monoculturas em paisagens homogêneas. Por exemplo, se ao invés de termos duas paisagens com 30\% de vegetação nativa, tivermos uma de 50 e outra de $10 \%$, a paisagem de $10 \%$ será formada unicamente por fragmentos muito isolados, e poderá ser uma importante barreira para movimentação das espécies em escala regional. Esta situação não é desejável em termos biológicos, nem em termos econômicos, uma vez que as RL têm importante papel no funcionamento da paisagem. Em particular, as RL propiciam importantes serviços ambientais, como o controle de pragas, e aumento da polinização e da produtividade de algumas culturas (De Marco \& Coelho 2004). Ademais, são as RL que permitem que a cobertura de vegetação nativa da paisagem fique acima dos limiares ecológicos citados anteriormente, protegendo assim parte da biota nativa, e favorecendo os fluxos biológicos entre Unidades de Conservação. Mesmo fragmentos muito pequenos podem ser importantes neste sentido. O exemplo mais claro é o da Mata Atlântica, onde fragmentos com menos de 50 ha representam um terço da cobertura florestal do bioma, e desempenham papel fundamental na redução do isolamento entre grandes fragmentos (Ribeiro et al. 2009).

Desta forma, o "regime de condomínio" é salutar para a manutenção ou a criação de grandes fragmentos, formados pela agregação de diversas RL particulares, porém é necessária a existência de um mecanismo que limite o uso deste recurso, para não criar paisagens depauperadas de vegetação, principalmente em áreas planas, onde as APP são também menos extensas. É possível estabelecer um limite percentual de áreas de RL em condomínio, e/ou limitar o uso deste mecanismo a bacias hidrográficas de extensão geográfica intermediárias, da ordem de 10 a 50 mil ha. Nesta situação, há menores chances de haver grandes disparidades de cobertura vegetacional, e ao mesmo tempo aumenta a representatividade da heterogeneidade ambiental, criando-se assim redes de RL biologicamente complementares, com maior diversidade biológica.

\section{Conclusões}

Contrariamente ao que se tem dito, o estado das pesquisas atuais oferece forte sustentação para critérios e parâmetros definidos pelo Código Florestal, sendo que em alguns casos haveria necessidade de expansão da área de conservação definida por esses critérios, em particular na definição das Áreas de Preservação Permanente. A literatura científica levantada mostra ainda que as recentes propostas de alteração deste Código, em particular alterando a extensão ou as regras de uso das Reservas Legais, podem trazer graves prejuízos ao patrimônio biológico e genético brasileiro. Os dados aqui apresentados, que retratam avanços recentes da ciência na área de ecologia e conservação, deveriam ser considerados em qualquer discussão sobre modificação do Código Florestal, e na procura da melhor configuração de nossas paisagens, que permita maximizar os serviços ecossistêmicos e o potencial de conservação da biodiversidade da biota nativa, sem prejudicar o desenvolvimento econômico nacional.

\section{Agradecimentos}

Agradeço as edições e sugestões prestadas por Roberto Varjabedian e Alexandre Igari numa versão preliminar deste artigo.

\section{Referências}

Almeida Vieira F \& De Carvalho D, 2008. Genetic structure of an insect-pollinated and bird-dispersed tropical tree in vegetation fragments and corridors: Implications for conservation. Biodiversity and Conservation, 17:2305-2321.

Andrén H, 1994. Effects of habitat fragmentation on birds and mammals in landscapes with different proportions of suitable habitat: a review. Oikos, 71:355-366.

Awade M \& Metzger JP, 2008. Using gap-crossing capacity to evaluate functional connectivity of two Atlantic rainforest birds and their response to fragmentation. Austral Ecology, 33:863-871.

Barlow J. et al., 2007a. Quantifying the biodiversity value of tropical primary, secondary, and plantation forests. Proceedings of the National Academy of Sciences of the United States of America, 104:18555-18560.

Barlow J et al., 2007b. The value of primary, secondary and plantation forests for Amazonian birds. Biological Conservation, 136:212-231.

Boscolo D et al., 2008. Importance of inter-habitat gaps and stepping-stones for lesser woodcreepers (Xiphorhynchus fuscus) in the Atlantic Forest, Brazil. Biotropica, 40:273-276.

De Marco Jr. P \& Coelho FM, 2004. Services performed by the ecosystem: Forest remnants influence agricultural cultures' pollination and production. Biodiversity and Conservation, 13:1245-1255.

Develey PF \& Stouffer PC, 2001. Effects of roads on movements by understory birds in mixed-species flocks in Central Amazonian Brazil. Conservation Biology, 15:1416-1422.

Develey PF \& Metzger JP, 2006. Emerging threats to birds in Brazilian Atlantic forests: the roles of forest loss and configuration in a severely fragmented ecosystem. In Laurance WF and Peres CA. (eds.). Emerging Threats to Tropical Forests. Chicago: University of Chicago Press. p. 269-290.

Diamond JM, 1975. The island dilemma: lessons of modern biogeographic studies for design of natural reserves. Biological Conservation, 7:129-145. 
Diamond JM, 1976. Island biogeography and conservation: strategy and limitations. Science, 193:1027-1029.

Fahrig L, 2003. Effects of habitat fragmentation on biodiversity. Annual Review of Ecology, Evolution and Systematic, 34:487-515.

Faria D et al., 2006. Bat and bird assemblages from forests and shade cacao plantations in two contrasting landscapes in the Atlantic Forest of southern Bahia, Brazil. Biodiversity and Conservation, 15:587-612.

Faria D et al., 2007. Ferns, frogs, lizards, birds and bats in forest fragments and shade cacao plantations in two contrasting landscapes in the Atlantic forest, Brazil. Biodiversity and Conservation, 16:2335-2357.

Fonseca CR et al., 2009. Towards an ecologically sustainable forestry in the Atlantic Forest. Biological Conservation, 142:1209-1219.

Keuroghlian A \& Eaton DP, 2008. Importance of rare habitats and riparian zones in a tropical forest fragment: Preferential use by Tayassu pecari, a wide-ranging frugivore. Journal of Zoology, 275:283-293.

Laurance SG \& Laurance WF, 1999. Tropical wildlife corridors: Use of linear rainforest remnants by arboreal mammals. Biological Conservation, 91:231-239.

Laurance WF et al., 2002. Ecosystem decay of Amazonian forest fragments: a 22-year investigation. Conservation Biology, 16:605-618.

Lees AC \& Peres CA, 2008. Conservation value of remnant riparian forest corridors of varying quality for Amazonian birds and mammals. Conservation Biology, 22:439-449.

Lima MG \& Gascon C, 1999. The conservation value of linear forest remnants in central Amazonia. Biological Conservation, 91:241-247.

Lindenmayer DB \& Luck G, 2005. Synthesis: Thresholds in conservation and management. Biological Conservation, 124:351-354.

Lopes AV et al., 2009. Long-term erosion of tree reproductive trait diversity in edge-dominated Atlantic forest fragments. Biological Conservation, 142:1154-1165.

Maltchik L et al., 2008. Dynamics of the terrestrial amphibian assemblage in a flooded riparian forest fragment in a Neotropical region in the south of Brazil. Brazilian Journal of Biology, 68:763-769.

Marinho-Filho J \& Verissimo EW, 1997. The rediscovery of Callicebus personatus barbarabrownae in northeastern Brazil with a new western limit for its distribution. Primates, 38:429-433.

Marini MA et al., 2009. Predicted climate-driven bird distribution changes and forecasted conservation conflicts in a neotropical savanna. Conservation Biology, 23:1558-1567.

Martensen AC, Pimentel RG \& Metzger JP, 2008. Relative effects of fragment size and connectivity on bird community in the Atlantic Rain Forest: Implications for conservation. Biological Conservation, 141:2184-2192.

Metzger JP, 2001. Effects of deforestation pattern and private nature reserves on the forest conservation in settlement areas of the Brazilian Amazon. Biota Neotropica. Avaliable from: http://www.biotaneotropica.org.br/v1n12.

Metzger JP, 2002. Bases biológicas para definição de Reservas Legais. Ciência Hoje, 31:183-184.

Metzger JP \& Décamps H, 1997. The structural connectivity threshold: an hypothesis in conservation biology at the landscape scale. Acta Ecologica, 18:1-12.

Metzger JP, Bernacci LC \& Goldenberg R, 1997. Pattern of tree species diversity in riparian forest fragments with different widths (SE Brazil). Plant Ecology, 133:135-152.

Metzger JP, Goldenberg R \& Bernacci LC, 1998. Diversidade e estrutura de fragmentos de mata de várzea e de mata mesófila semidecídua submontana do rio Jacaré-Pepira (SP). Revista Brasileira de Botânica, 21:321-330.

Metzger JP et al., 2009. Time-lag in biological responses to landscape changes in a highly dynamic Atlantic forest region. Biological Conservation, 142:1166-1177.

Michalski F et al., 2006. Human-wildlife conflicts in a fragmented Amazonian forest landscape: Determinants of large felid depredation on livestock. Animal Conservation, 9:179-188.

Miranda EE et al., 2008. Alcance Territorial da Legislação Ambiental e Indigenista. Campinas: Embrapa Monitoramento por Satélite. [cited 2010 March 8]. Available from:http:// www.alcance.cnpm.embrapa.br.Acesso em: 8 mar. 2010.

Moura DC \& Schlindwein C, 2009. The gallery forests of the São Francisco river as corridors for euglossine bees (Hymenoptera: Apidae) from tropical rainforests. Neotropical Entomology, 38:281-284.

Oliveira-Filho FJB \& Metzger JP, 2006. Thresholds in landscape structure for three common deforestation patterns in the Brazilian Amazon. Landscape Ecology, 21:1061-1073.

Oliveira-Filho AT et al., 1994a. Differentiation of streamside and upland vegetation in an area of montane semideciduous forest in southeastern brazil. Flora,189:287-305.

Oliveira-Filho AT et al., 1994b. Effects of soils and topography on the distribution of tree species in a tropical riverine forest in south-eastern Brazil. Journal of Tropical Ecology, 10:483-508.

Pardini R et al., 2005. The role of forest structure, fragment size and corridors in maintaining small mammal abundance and diversity in an Atlantic forest landscape. Biological Conservation, 124:253-266.

Pardini R et al., 2009. The challenge of maintaining Atlantic forest biodiversity: a multi-taxa conservation assessment of specialist and generalist species in an agro-forestry mosaic in southern Bahia. Biological Conservation, 142:1178-1190.

Pinay G \& Décamps H, 1988. The role of riparian woods in regulating nitrogen fluxes between the alluvial aquifer and surface water: a conceptual model. Regulated Rivers - Research \& Management, 2:507-516.

Quigley HB \& Crawshaw J, 1992. A conservation plan for the jaguar Panthera onca in the Pantanal region of Brazil. Biological Conservation, 61:149-157. 
Ribeiro MC et al., 2009. Brazilian Atlantic forest: how much is left and how is the remaining forest distributed? Implications for conservation. Biological Conservation, 142:1141-1153

Rodrigues RR \& Leitão-Filho HF, 2004. Matas Ciliares: Conservação e Recuperação. 3 ed. São Paulo: EDUSP/ FAPESP.

Santos BA et al., 2008. Drastic erosion in functional attributes of tree assemblages in Atlantic forest fragments of northeastern Brazil. Biological Conservation, 141:249-260.

Shaffer M, 1987. Minimum viable populations: coping with uncertainty. In Soulé ME (ed.). Viable Populations for Conservation. Cambridge: Cambridge University Press. p. 69-86.

Simberloff D \& Abele LG, 1976. Island biogeography theory and conservation practice. Science, 191:285-286.

Simberloff D \& Abele LG, 1982. Refuge design and island biogeography theory: effects of fragmentation. American Naturalist, 120:41-50.
Soulé ME \& Simberloff D, 1986. What do genetics and ecology tell us about the design of nature reserves? Biological Conservation, 35:19-40.

Stauffer D, 1985. Introduction to percolation theory. London: Taylor \& Francis.

Tubelis DP, Cowling A \& Donnelly C, 2004. Landscape supplementation in adjacent savannas and its implications for the design of corridors for forest birds in the central Cerrado, Brazil. Biological Conservation, 118:353-364.

Uezu A, Metzger JP \& Vielliard JM, 2005. Effects of structural and functional connectivity and patch size on the abundance of seven Atlantic Forest bird species. Biological Conservation, 123:507-519.

Recebido: Março 2010

Primeira Decisão: Março 2010

Aceito: Março 2010 